\title{
Characterization and phylogenetic affiliation of Actinobacteria from tropical soils with potential uses for agro-industrial processes
}

\author{
J.C.M. Dornelas ${ }^{1}$, J.E.F. Figueiredo ${ }^{2}$, C.S. de Abreu ${ }^{3}$, U.G.P. Lana ${ }^{2}$, \\ C.A. Oliveira ${ }^{2}$ and I.E. Marriel ${ }^{1,2}$ \\ ${ }^{1}$ Programa de Pós-Graduação em Ciências Agrárias, \\ Departamento de Ciências Agrárias, Universidade Federal de São João Del Rei, \\ Sete Lagoas, MG, Brasil \\ ${ }^{2}$ Laboratório de Microbiologia e Biologia Molecular, Embrapa Milho e Sorgo, \\ Sete Lagoas, MG, Brasil \\ ${ }^{3}$ Programa de Pós-Graduação em Microbiologia, Departamento de Microbiologia, \\ Universidade Federal de Minas Gerais, Belo Horizonte, MG, Brasil \\ Corresponding author: I.E. Marriel \\ E-mail: ivanildo.marriel@embrapa.br \\ Genet. Mol. Res. 16 (3): gmr16039703 \\ Received April 17, 2017 \\ Accepted July 3, 2017 \\ Published August 31, 2017 \\ DOI http://dx.doi.org/10.4238/gmr16039703
}

Copyright (C) 2017 The Authors. This is an open-access article distributed under the terms of the Creative Commons Attribution ShareAlike (CC BY-SA) 4.0 License.

\begin{abstract}
Secondary metabolites produced by Actinobacteria of tropical soils represent a largely understudied source of novel molecules with relevant application in medicine, pharmaceutical and food industries, agriculture, and environmental bioremediation. The present study aimed to characterize sixty-nine Actinobacteria isolated from compost and tropical soils using morphological, biochemical, and molecular methods. All the isolates showed high variation for morphological traits considering the color of pigments of the aerial and vegetative mycelium and spore chain morphology. The enzymatic activity of amylase, cellulase, and lipase was highly variable. The amylase activity was detected in $53(76.81 \%)$ isolates. Eighteen
\end{abstract}

Genetics and Molecular Research 16 (3): gmr16039703 
isolates showed enzymatic index (EI) $>4.0$, and the isolates ACJ 45 (Streptomyces curacoi) and ACSL 6 (S. hygroscopicus) showed the highest EI values (6.44 and 6.42, respectively). The cellulase activity varied significantly $(\mathrm{P} \leq 0.05)$ among the isolates. Twenty-nine isolates $(42.02 \%)$ showed high cellulase activity, and the isolates ACJ 48 (S. chiangmaiensis) and ACJ 53 (S. cyslabdanicus) showed the highest EI values (6.56 for both isolates). The lipase activity varied statistically $(\mathrm{P} \leq 0.05)$ with fourteen isolates $(20.29 \%)$ considered good lipase producers (EI > 2.0). The isolate ACSL 6 (S. hygroscopicus) showed the highest EI value of 2.60. Molecular analysis of partial 16S rRNA gene sequencing revealed the existence of 49 species, being 38 species with only one representative member and 11 species represented by one or more strains. All species belonged to three genera, namely Streptomyces (82.61\%), Amycolatopsis (7.25\%), and Kitasatospora (10.14\%). The present results showed the high biotechnological potential of different Actinobacteria from tropical soils.

Key words: Actinomycetes; Morphological traits; Enzymatic activity; $16 \mathrm{~S}$ rRNA gene; Molecular identification

\section{INTRODUCTION}

The phylum Actinobacteria (Actinomycetes) is an ancient bacterial group branched off from the other prokaryotes very early in the evolutionary process (Battistuzzi et al., 2004; Ventura et al., 2007). Actinobacteria comprise an ecologically diverse group ubiquitously distributed in various natural environments as free-living, pathogens, and endophyte symbionts (Hardoim et al., 2015). They are distinguished as Gram-positive bacteria, normally aerobic, non-acid fast and with a high GC content in their DNA, varying to less than $50 \%$ in a few species to more than $70 \%$ in some genera (Lewin et al., 2016).

Actinobacteria exhibit a high level of diversity of biochemical features, such as the production of a wide variety of secondary metabolites and extracellular enzymes with relevant applications in different fields (Suneetha et al., 2011; Barka et al., 2015; Li et al., 2016). Although near half of the bioactive molecules with different uses in medicine, industrial processes, agriculture, and environmental bioremediation are produced by Actinobacteria, it represents only a small fraction of the overall metabolites already identified in this bacterial group (Abid et al., 2016). New enzymes selected based on enzymatic index (EI) criterion have been extensively explored commercially by the food, textile, and biofuel industry. Amylase, lipase, cellulase are some examples of enzymes isolated from Actinobacteria and currently used in the global market (Sathya and Ushadevi, 2014). Thus, the understudied Actinobacteria from tropical soils may represent a promising new source of secondary metabolites for many purposes.

The taxonomy of Actinobacteria has been a subject of intense debate. Traditionally, morphological traits as growth pattern and mycelia type are the main characteristics used to define order, genera, and species in this group, and biochemical tests using enzyme activities are used for identifying new bioactive metabolites. Recently, molecular data based on DNA sequencing of the 16S rRNA gene assumed an important role on systematic of the phylum Actinobacteria (Ventura et al., 2007).

Genetics and Molecular Research 16 (3): gmr16039703 
In the present study, morphological traits, enzymatic activities, and partial sequencing of the 16S rRNA gene were used for characterizing Actinobacteria isolated from Brazilian tropical soils.

\section{MATERIAL AND METHODS}

The study was performed with sixty-nine bacterial isolates deposited at the Embrapa Maize and Sorghum Multifunctional Culture Collection (CCMF-CNPMS) that were previously identified as Actinobacteria based on their growth morphology (Table 1).

\begin{tabular}{|c|c|c|c|}
\hline Origin & Location site & Year & Identification \\
\hline Cerrado soil with high level of phosphate at the Experimental Station of Embrapa & Sete Lagoas & 2000 & ACSL $1 \mathrm{~A}, 2,8$ \\
\hline Cerrado soil from the Experimental Station of Embrapa & Sete Lagoas & 2001 & ACSL 12, 16A \\
\hline Cerrado soil from the Experimental Station of Embrapa & Sete Lagoas & 2004 & ACSL $485,490,495,509,517$ \\
\hline Cerrado soil from the Experimental Station of Embrapa & Sete Lagoas & 2014 & ACSL 1B, $6,13,16 \mathrm{~B}, 18 \mathrm{~B}, 22,23,27 \mathrm{~B}$ \\
\hline Maize Rhizospheral soil from the Experimental Station of Embrapa & Sete Lagoas & 2004 & ACSL $7,18 \mathrm{~A}, 25,27 \mathrm{~A}, 50,53,54,64 \mathrm{~B}, 67,77,82,91$ \\
\hline Organic farming from the Experimental Station of Embrapa & Sete Lagoas & 2002 & ACSL $432,448,449,450,453,457,470$ \\
\hline Cerrado soil planted with eucalyptus & Sete Lagoas & 2001 & ACSL $64 \mathrm{~A}, 80,83,85,93,115$ \\
\hline Cerrado soil planted with eucalyptus and pinus woods & Sete Lagoas & 2003 & ACSL 404 \\
\hline Cerrado soil from the Fazenda Santa Rita Experimental Station & Prudente de Morais & 2006 & ACPM 641 \\
\hline Cerrado soil from the Fazenda Santa Rita Experimental Station & Prudente de Morais & 2007 & ACPM $5,29,31,38,66$ \\
\hline Cerrado soil planted with peanuts from the Fazenda Santa Rita Experimental Station & Prudente de Morais & 2002 & ACPM $346,363,364$ \\
\hline Agricultural Cerrado soil from the Experimental Station of Embrapa & Jaíba & 2001 & ACJ 66,76 \\
\hline Cerrado degradated soil & Jaíba & 2001 & ACJ $1,17,26,29$ \\
\hline Mata seca & Jaíba & 2001 & $\mathrm{ACJ} 36,43,45$ \\
\hline Protected area & Jaiba & 2001 & ACJ $48,49,51,52,53$ \\
\hline Compost & Papagaios & 2013 & ACP 35 \\
\hline Compost & Capim Branco & 2015 & ACCB 1 \\
\hline
\end{tabular}

The isolates were grown in the agar glycerol-asparagine (AGA) medium $[1 \mathrm{~g} / \mathrm{L}$ L-asparagine, $10 \mathrm{~g} / \mathrm{L}$ glycerol, $1 \mathrm{~g} / \mathrm{L} \mathrm{KH}_{2} \mathrm{PO}_{4}, 15 \mathrm{~g} / \mathrm{L}$ agar, $1 \mathrm{~mL} / \mathrm{L}$ micronutrient solution $\left(0.1 \mathrm{~g} \mathrm{FeSO}_{4} \times 7 \mathrm{H}_{2} \mathrm{O}, 0.1 \mathrm{~g} \mathrm{MnCl} \times 4 \mathrm{H}_{2} \mathrm{O}, 0.1 \mathrm{~g} \mathrm{ZnSO}_{4} \times 7 \mathrm{H}_{2} \mathrm{O}\right.$, qsp $100 \mathrm{~mL}$ deionized water $\left.)\right]$ according to Pridham and Lyons (1961) and supplemented with $0.03 \mathrm{~g} / \mathrm{L}$ cycloheximide. After inoculations, the plates were incubated for 14 days at $28^{\circ} \mathrm{C}$.

\section{Morphological characterization}

The morphological analysis was performed according to Shirling and Gottlieb (1966). The following growing cultural parameters were evaluated: the color of the vegetative and aerial mycelium, and changes in the color of the medium around the colony.

For the micromorphological analysis, the microculture technique was used according to Holt et al. (1994). After the incubation period, the coverslip was removed and placed on another sterile microscopic slide containing $10 \mu \mathrm{L}$ Amann lactophenol and the edges were sealed with colorless nail enamel. The spore chain morphology was observed under the optical microscope Olympus BX 60 (Olympus Optical Co. Ltd., Tokyo, Japan) with 1000X magnification, and photographed with a digital camera (Leica DFC 490, Leica Microsystems Inc., Buffalo Grove, IL, USA).

\section{Molecular characterization}

The DNA extraction, PCR, and DNA sequencing were made according to Lana et al. (2012). The 16S rRNA gene was amplified using the universal primers 8F (5'-AGA GTT

Genetics and Molecular Research 16 (3): gmr16039703 
TGA TCC TGG CTC AG-3') and 1492R (5'-GGT TAC CTT GTT ACG ACT T-3') (Turner et al., 1999). The sequencing was made with the PCR primers and the internal primers $515 \mathrm{~F}$ (5'GTG CCA GCM GCC GCG GTA A-3'; Turner et al., 1999) and 902R (5'-GTC AAT TCI TTT GAG TTT YAR YC-3'; Hodkinson and Lutzoni, 2009). In the primers, the letters Y; M; R; and I represent the nucleotides cytosine or thymine; adenine or cytosine; adenine or guanine; and a modified guanine, respectively.

The DNA sequences were generated in the Applied Biosystems 3500xL Genetic Analyzer (Applied Biosystem, Foster City, CA, USA). The nucleotide sequences were edited using the Sequencher 4.1 program and compared in the GenBank database (http://www.ncbi. nlm.nih.gov/) through the BLAST N program (Altschul et al., 1997) located at the NCBI (National Center for Biotechnology Information).

\section{Phylogenetic analysis}

The phylogenetic analysis was performed by the neighbor-joining method using the Molecular Evolutionary Genetics Analysis version 5 (MEGA5) program (Tamura et al., 2011).

\section{Enzymatic activity}

To test the enzymatic activity of amylase, cellulase, and lipase, disks of cultures on agar plate were inoculated in triplicate in a completely random design and incubated at $28^{\circ} \mathrm{C}$ for 10 days (amylase and cellulase) and $72 \mathrm{~h}$ (lipase). The EI was estimated by the following equation: $\mathrm{EI}=$ ratio of halo diameter/ratio of colony diameter. The isolates were classified as non-producer $(\mathrm{EI}=0)$, low producer $(0>\mathrm{EI} \leq 2)$, middle producer $(2>\mathrm{EI} \leq 4)$, and high producer $(\mathrm{EI}>4)$. The data were analyzed for significance $(\mathrm{P}<0.05)$ using the Scott-Knott test.

\section{Amylase}

The amylase production was determined as described by Coon et al. (1957) modified by the addition of $6.6 \%$ soluble starch. The isolates were inoculated on the starch agar medium $(6.6 \mathrm{~g} / \mathrm{L}$ soluble starch, $0.5 \mathrm{~g} / \mathrm{L}$ sodium chloride, $3 \mathrm{~g} / \mathrm{L}$ meat extract, $1 \mathrm{~g} / \mathrm{L}$ peptone casein, $15 \mathrm{~g} / \mathrm{L}$ agar with the $\mathrm{pH}$ adjusted to 7.0). After culture growth, $10 \mathrm{~mL}$ Lugol's solution $(5 \mathrm{~g}$ iodine, $10 \mathrm{~g}$ potassium iodide in $100 \mathrm{~mL}$ distilled water) was diluted to $1: 10$ and added to the dishes. The amylase production was detected by the formation of a light yellow zone around the colony corresponding to the discoloration of the medium.

\section{Cellulase}

The cellulase production was tested in the culture medium supplemented with carboxymethylcellulose (CMC) as the sole carbon source $\left(3 \mathrm{~g} / \mathrm{L} \mathrm{NaNO}_{3}, 1 \mathrm{~g} / \mathrm{L} \mathrm{K}_{2} \mathrm{HPO}_{4}, 0.5\right.$ $\mathrm{g} / \mathrm{L} \mathrm{MgSO}_{4}, 0.5 \mathrm{~g} / \mathrm{L} \mathrm{KCl}, 10 \mathrm{mg} / \mathrm{L} \mathrm{FeSO}_{4} .7 \mathrm{H}_{2} \mathrm{O}, 10 \mathrm{~g} / \mathrm{L} \mathrm{CMC}, 15 \mathrm{~g} / \mathrm{L}$ agar with the $\mathrm{pH}$ adjusted to 7.0 ) according to Lewis (1988). After incubation, $10 \mathrm{~mL} 0.5 \%$ Congo red dye was added to each plate, incubated for $15 \mathrm{~min}$ at room temperature and washed with $\mathrm{NaCl}(5 \mathrm{M})$. Afterward, the excess of solution was discarded, and $10 \mathrm{~mL} \mathrm{NaCl}$ solution $(1 \mathrm{M})$ was added to each plate and incubated for $30 \mathrm{~min}$ at room temperature. The production of the enzyme was observed by the discoloration of the medium, which forms an orange zone around the colony.

Genetics and Molecular Research 16 (3): gmr16039703 


\section{Lipase}

Lipase production was tested according to Savitha et al. (2007) using a culture medium with the following composition: $5 \mathrm{~g} / \mathrm{L}$ peptone, $1 \mathrm{~g} / \mathrm{L}$ yeast extract, $4 \mathrm{~g} / \mathrm{L}$ sodium chloride, 15 $\mathrm{g} / \mathrm{L}$ agar, $31.25 \mathrm{~mL} / \mathrm{L}$ olive oil, $0.01 \mathrm{~g} / \mathrm{L}$ Rhodamine $\mathrm{B}, \mathrm{pH}$ 7.0. Disks of the cultured isolates were inoculated in the medium, and after the incubation period, the plates were exposed to ultraviolet radiation to observe the formation of blue stained halos around the positive colonies, which is the parameter used to indicate the activity of the enzyme (Colen, 2006).

\section{RESULTS}

In this study, the analysis of morphological traits, enzyme activities, and molecular sequencing revealed a high level of variability among sixty-nine isolates of Actinobacteria from composts and tropical soils.

\section{Morphological characterization}

The analyses of the four main morphological traits used for Actinobacteria identification were highly variable (Table 2). Besides these characteristics, the production of classic pulverulent mycelium and radial growth confirmed the identity of the isolates as Actinobacteria. All sixty-nine morphospecies produced vegetative mycelium on the AGA medium with evident variation in the pattern of development, color, and pigment production (Figure 1). The release of soluble pigments on the AGA medium was observed in $50.72 \%$ of the isolates and varied from yellow (28.57\%), light brown (40\%), and dark brown (28.57\%) to black $(2.86 \%)$.

The micromorphological features of the bacterial colonies were assessed with a light microscope to determine the presence, absence, and morphology of the spore chain compared with valid criteria reported in the Bergey's Manual of Systematic Bacteriology (Holt et al., 1994) (Table 2). The morphological structure of the spore chain varied depending on the isolate, and was classified as straight (10.14\%), retinaculum apertum (28.99\%), spiral (27.54\%), and flexuous (33.33\%) (Figure 2). The phylogenetic tree constructed using morphological and biochemical data showed all species grouped together (data not shown).

\section{Molecular characterization and phylogenetic analysis}

Partial sequences of the 16S rRNA gene, ranging in length from 1210 to 1400 nucleotides, were determined for 69 isolates. The molecular characterization based on nucleotide comparisons of the 16S rRNA gene with nucleotide sequences deposited in the GenBank (accession numbers: KY585931 to KY585999) confirmed the morphological identity of the isolates as Actinobacteria (Table 3). A total of forty-nine taxa were identified distributed among the following three genera: Streptomyces (82.61\%), Amycolatopsis $(10.14 \%)$, and Kitasatospora $(7.25 \%)$.

The phylogenetic tree constructed by the maximum likelihood, evolutionary distance, and maximum parsimony methods with the MEGA5 program (Tamura et al., 2011) generated two distinct clades with Amycolatopsis separated from Streptomyces and Kitasatospora (Figure 3). The clade formed by the genera Streptomyces and Kitasatospora was divided 
J.C.M. Dornelas et al.

Table 2. Morphological characteristics of the Actinobacteria isolates from composts and tropical soils from different collecting places of Brazil.

\begin{tabular}{|c|c|c|c|c|c|c|}
\hline \multirow[t]{3}{*}{ Isolate } & \multicolumn{5}{|c|}{ Macromorphology } & \multirow{3}{*}{$\begin{array}{l}\text { Micromorphology } \\
\text { Spore chain type }\end{array}$} \\
\hline & \multicolumn{3}{|c|}{ Aerial mycelium } & \multirow{2}{*}{ Vegetative mycelium } & \multirow[t]{2}{*}{ Pigments } & \\
\hline & Center & Edge & Uniform & & & \\
\hline ACSL 1A & Yellow & Gray & & Beige & - & Retinaculum apertum \\
\hline ACSL 1B & & & White & Black & Black & Retinaculum apertum \\
\hline ACSL 2 & Cream & Yellow & & Cream & - & Spiral \\
\hline ACSL 6 & & & Dark brown & Dark brown & - & Retinaculum apertum \\
\hline ACSL 7 & Beige & White & & Cream & - & Spiral \\
\hline ACSL 8 & Beige & White & & Beige & - & Flexuous \\
\hline ACSL 12 & & & Light brown & Dark brown & Light brown & Flexuous \\
\hline ACSL 13 & & & Dark brown & Dark brown & - & Retinaculum apertum \\
\hline ACSL 16A & & & Cream & Cream & - & Straight \\
\hline ACSL 16B & Beige & White & & Cream & - & Spiral \\
\hline ACSL 18A & & & Cream & Cream & Yellow & Retinaculum apertum \\
\hline ACSL 18B & & & Gray & Dark brown & Dark brown & Straight \\
\hline ACSL 22 & & & White & Dark brown & Yellow & Flexuous \\
\hline ACSL 23 & White & Gray & & Light brown & Light brown & Flexuous \\
\hline ACSL 25 & & & Cream & Light brown & - & Flexuous \\
\hline ACSL 27A & & & Cream & Cream & - & Spiral \\
\hline ACSL 27B & & & Gray & Beige & Light brown & Retinaculum apertum \\
\hline ACSL 50 & & & Brown & Brown & Dark brown & Flexuous \\
\hline ACSL 53 & & & Beige & Beige & - & Spiral \\
\hline ACSL 54 & & & Ocher & Ocher & - & Flexuous \\
\hline ACSL 64A & & & Beige & Beige & Light brown & Spiral \\
\hline ACSL 64B & & & Light brown & Light brown & Yellow & Retinaculum apertum \\
\hline ACSL 67 & Brown & Gray & & Dark brown & Light brown & Flexuous \\
\hline ACSL 77 & & & Light brown & Light brown & Light brown & Flexuous \\
\hline ACSL 80 & & & Gray & Dark brown & Dark brown & Straight \\
\hline ACSL 82 & & & Beige & Beige & - & Spiral \\
\hline ACSL 83 & & & Cream & Cream & Yellow & Retinaculum apertum \\
\hline ACSL 85 & & & Gray & Dark brown & Dark brown & Straight \\
\hline ACSL 91 & & & White & Dark brown & Yellow & Flexuous \\
\hline ACSL 93 & Dark gray & Brown/purple & & Dark brown & - & Retinaculum apertum \\
\hline ACSL 115 & & & White & Light brown & Light brown & Spiral \\
\hline ACSL 404 & Cream & Yellow & & Cream & - & Spiral \\
\hline ACSL 432 & Dark brown & Gray & & Brown & Light brown & Spiral \\
\hline ACSL 448 & & & White & Beige & Light brown & Spiral \\
\hline ACSL 449 & & & White & Dark brown & Yellow & Flexuous \\
\hline ACSL 450 & & & Cream & Cream & Yellow & Flexuous \\
\hline ACSL 453 & Beige & White & & Cream & - & Spiral \\
\hline ACSL 457 & & & Beige & Beige & - & Flexuous \\
\hline ACSL 470 & Light brown & Gray & & Brown & - & Flexuous \\
\hline ACSL 485 & Dark brown & Light brown & & Brown & Dark brown & Retinaculum apertum \\
\hline ACSL 490 & Yellow & White & & Cream & - & Flexuous \\
\hline ACSL 495 & Yellow & Gray & & Beige & - & Flexuous \\
\hline ACSL 509 & Beige & Gray & & White & - & Retinaculum apertum \\
\hline ACSL 517 & & & Beige & Beige & - & Straight \\
\hline ACPM 5 & & & Gray & Light brown & - & Spiral \\
\hline ACPM 29 & & & Dark brown & Dark brown & Light brown & Retinaculum apertum \\
\hline ACPM 31 & Dark brown & Light brown & & Dark brown & Light brown & Flexuous \\
\hline ACPM 38 & White & Gray & & Beige & - & Flexuous \\
\hline ACPM 66 & Gray & White & & Light brown & Dark brown & Flexuous \\
\hline ACPM 346 & & & Ocher & Ocher & - & Straight \\
\hline ACPM 363 & Beige & White & & Cream & - & Spiral \\
\hline ACPM 364 & & & Gray & Dark brown & - & Flexuous \\
\hline ACPM 641 & & & Beige & Light brown & Light brown & Retinaculum apertum \\
\hline ACJ 1 & Beige & White & & White & - & Retinaculum apertum \\
\hline ACJ 17 & White & Pink & & Beige/pink & - & Spiral \\
\hline ACJ 26 & & & White & Ocher & Yellow & Spiral \\
\hline ACJ 29 & Beige & White & & Cream & - & Spiral \\
\hline ACJ 36 & Dark brown & Light brown & & Brown & Dark brown & Retinaculum apertum \\
\hline ACJ 43 & Dark brown & Light brown & & Brown & Dark brown & Retinaculum apertum \\
\hline ACJ 45 & & & Light brown & Light brown & Light brown & Spiral \\
\hline ACJ 48 & & & Brown & Brown & Dark brown & Retinaculum apertum \\
\hline ACJ 49 & Gray & White & & Brown & - & Retinaculum apertum \\
\hline ACJ 51 & & & Light brown & Light brown & Yellow & Retinaculum apertum \\
\hline ACJ 52 & Light brown & White & & Light brown & Light brown & Flexuous \\
\hline ACJ 53 & & & White & Cream & - & Retinaculum apertum \\
\hline ACJ 66 & Beige & White & & Cream & - & Spiral \\
\hline ACJ 76 & & & White & Dark brown & Yellow & Flexuous \\
\hline ACP 35 & Dark gray & Light gray & & Dark brown & Dark brown & Straight \\
\hline ACCB 1 & & & Cream & Cream & - & Flexuous \\
\hline
\end{tabular}

(-) indicate absence.

Genetics and Molecular Research 16 (3): gmr16039703 
into two separated subgroups. There was a clear separation between species, except for two Streptomyces strains grouped together within Kitasatospora. The branches were well supported with a bootstrap value above $70 \%$.

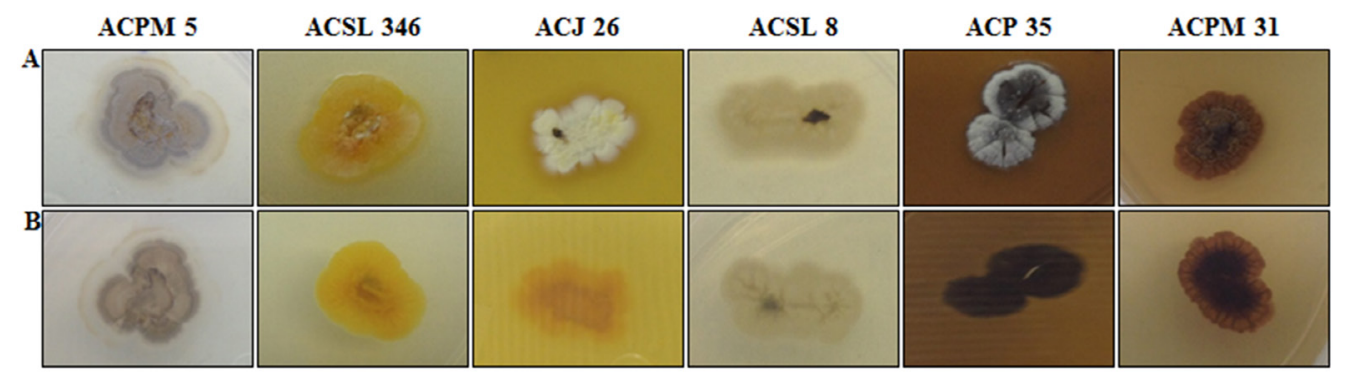

Figure 1. Morphological characteristics of representative Actinobacteria isolates from composts and tropical soils from different collecting places of Brazil. A. aerial mycelium; B. substrate mycelium.
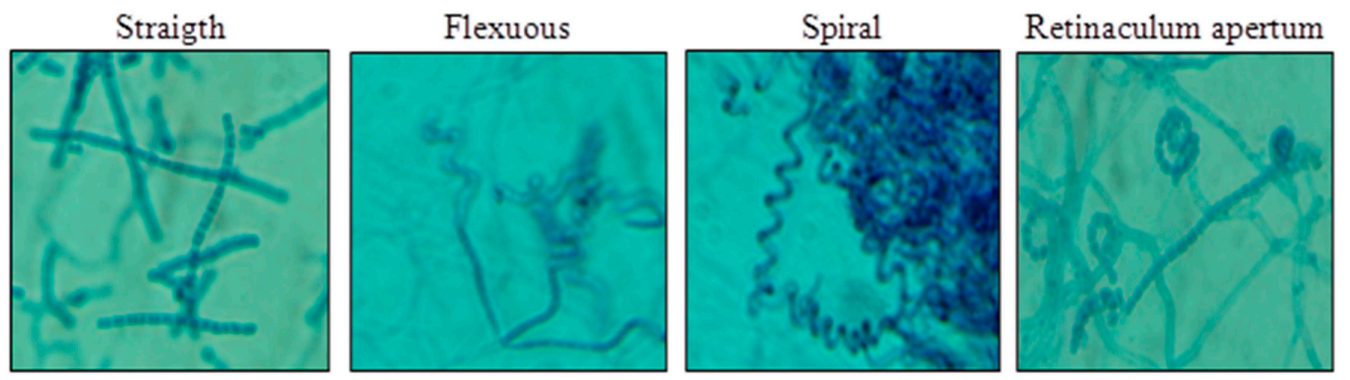

Figure 2. Spore chain morphology of Actinobacteria isolates from composts and tropical soils.

\section{Enzymatic activity}

The data of enzymatic activity for amylase, cellulase, and lipase are shown in Table 4. It was observed that $95.65 \%$ of the isolates were able to produce at least one of the three enzymes studied, even though they were not classified as good potential producers, that is, those that presented $E I \leq 2.0$. The production of amylase, cellulase, and lipase was observed in $76.81,79.71$, and $40.58 \%$ of the isolates, respectively. The isolates ACSL 93 (A. echigonensis), ACSL 76 (S. yunnanensis), and ACBB 1 (S. variabilis) did not show enzymatic activity (EI = 0 ) for any of the three enzymes tested (Table 4).

\section{Amylolytic activity}

Concerning amylase, the amylolytic activity was indicated by the presence of a yellow halo around the colonies (Figure 4a). The relationship between the halo and the colony diameters determined the EI. The EI was statistically significant $(\mathrm{P} \leq 0.05)$ among the isolates (Table 4). Sixteen isolates $(23.19 \%)$ did not produce a halo. Thus, they were considered nonamylase producers. All seven species identified as belonging to the genus Amycolatopsis $(10.4 \%)$ did not show amylolytic activity. 
Table 3. Molecular characterization of the Actinobacteria isolates from composts and tropical soils from different Brazilian collecting places.

\begin{tabular}{|c|c|c|c|c|c|c|c|}
\hline Isolate & $\begin{array}{c}\text { GenBank } \\
\text { accession No. }\end{array}$ & Species & Similarity (\%) & Isolate & $\begin{array}{c}\text { GenBank } \\
\text { accession No. }\end{array}$ & Species & Similarity (\%) \\
\hline ACSL 1A & KY585949 & Streptomyces seymenliensis & 99 & ACSL 450 & KY585946 & A. bullii & 98 \\
\hline ACSL 1B & KY585960 & S. massasporeus & 99 & ACSL 453 & KY585958 & S. galbus & 99 \\
\hline ACSL 2 & KY585933 & S. chartreusis & 99 & ACSL 457 & KY585981 & A. pretoriensis & 99 \\
\hline ACSL 6 & KY585976 & S. hygroscopicus & 100 & ACSL 470 & KY585944 & S. pseudovenezuelae & 100 \\
\hline ACSL 7 & KY585992 & S. galbus & 99 & ACSL 485 & KY585964 & S. psammoticus & 100 \\
\hline ACSL 8 & KY585974 & S. sporocinereus & 100 & ACSL 490 & KY585977 & A. kentuckyensis & 100 \\
\hline ACSL 12 & KY585963 & Kitasatospora atroaurantiaca & 99 & ACSL 495 & KY585979 & A. lexingtonensis & 99 \\
\hline ACSL 13 & KY585937 & S. hygroscopicus & 100 & ACSL 509 & KY585980 & S. deserti & 99 \\
\hline ACSL $16 \mathrm{~A}$ & KY585957 & S. purpeofuscus & 99 & ACSL 517 & KY585931 & S. phaeochromogenes & 98 \\
\hline ACSL 16B & KY585934 & S. galbus & 99 & ACPM 5 & KY585962 & S. olivochromogenes & 99 \\
\hline ACSL $18 \mathrm{~A}$ & KY585996 & S. longwoodensis & 99 & ACPM 29 & KY585969 & S. scabiei & 99 \\
\hline ACSL 18B & KY585951 & S. phaeochromogenes & 98 & ACPM 31 & KY585941 & S. phaeopurpureus & 99 \\
\hline ACSL 22 & KY585986 & S. yunnanensis & 99 & ACPM 38 & KY585942 & S. rishiriensis & 99 \\
\hline ACSL 23 & KY585972 & S. indiaensis & 100 & ACPM 66 & KY585984 & S. sioyaensis & 99 \\
\hline ACSL 25 & KY585988 & Amycolatopsis rifamycinica & 99 & ACPM 346 & KY585953 & S. endophyticus & 99 \\
\hline ACSL $27 \mathrm{~A}$ & KY585995 & S. lydicus & 99 & ACPM 363 & KY585940 & S. galbus & 99 \\
\hline ACSL 27B & KY585943 & S. corchorusii & 99 & ACPM 364 & KY585935 & K. viridis & 99 \\
\hline ACSL 50 & KY585998 & S. sampsonii & 99 & ACPM 641 & KY585959 & S. lannensis & 100 \\
\hline ACSL 53 & KY585990 & K. paracochleata & 99 & ACJ 1 & KY585945 & S. ossamyceticus & 100 \\
\hline ACSL 54 & KY585991 & S. sasae & 99 & ACJ 17 & KY585947 & S. bangladeshensis & 99 \\
\hline ACSL $64 \mathrm{~A}$ & KY585999 & S. coacervatus & 99 & ACJ 26 & KY585978 & S. сароатия & 99 \\
\hline ACSL 64B & KY585987 & S. griseoruber & 99 & ACJ 29 & KY585967 & S. galbus & 99 \\
\hline ACSL 67 & KY585994 & S. phaeopurpureus & 100 & ACJ 36 & KY585975 & S. psammoticus & 100 \\
\hline ACSL 77 & KY585997 & K. phosalacinea & 99 & ACJ 43 & KY585970 & S. psammoticus & 99 \\
\hline ACSL 80 & KY585938 & S. phaeochromogenes & 98 & ACJ 45 & KY585954 & S. curacoi & 99 \\
\hline ACSL 82 & KY585989 & K. paracochleata & 99 & ACJ 48 & KY585971 & S. chiangmaiensis & 100 \\
\hline ACSL 83 & KY585936 & S. longwoodensis & 99 & ACJ 49 & KY585948 & A. rhabdoformis & 100 \\
\hline ACSL 85 & KY585983 & S. phaeochromogenes & 99 & ACJ 51 & KY585968 & S. griseoruber & 100 \\
\hline ACSL 91 & KY585993 & S. yunnanensis & 99 & ACJ 52 & KY585973 & S. yaanensis & 100 \\
\hline ACSL 93 & KY585966 & A. echigonensis & 99 & ACJ 53 & KY585932 & S. cyslabdanicus & 99 \\
\hline ACSL 115 & KY585939 & S. thioluteus & 99 & ACJ 66 & KY585965 & S. galbus & 99 \\
\hline ACSL 404 & KY585950 & S. chartreusis & 100 & ACJ 76 & KY585955 & S. yunnanensis & 99 \\
\hline ACSL 432 & KY585961 & S. novaecaesareae & 99 & ACP 35 & KY585956 & S. laculatispora & 99 \\
\hline ACSL 448 & KY585952 & S. sioyaensis & 99 & ACCB 1 & KY585985 & S. variabilis & 100 \\
\hline ACSL 449 & KY585982 & S. yunnanensis & 99 & - & - & - & - \\
\hline
\end{tabular}

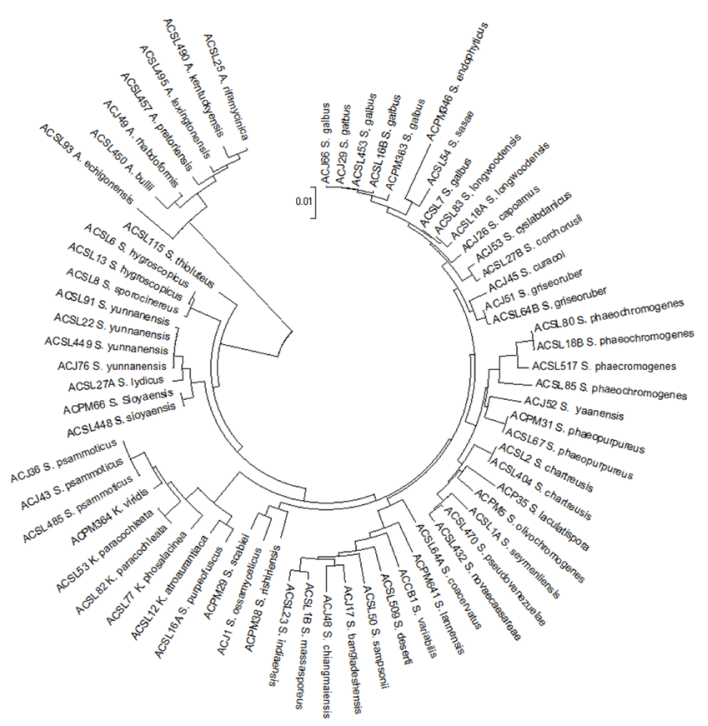

Figure 3. Dendrogram based on partial sequences of the 16S rRNA gene of the Actinobacteria isolates from composts and tropical soils.

Genetics and Molecular Research 16 (3): gmr16039703 
Table 4. Enzymatic index (EI) for amylase, cellulase, and lipase activity of the Actinobacteria isolates.

\begin{tabular}{|c|c|c|c|c|}
\hline \multirow[t]{2}{*}{ Isolate } & \multirow[t]{2}{*}{ Species } & \multicolumn{3}{|c|}{ Enzymatic index } \\
\hline & & Amylase & Cellulase & Lipase \\
\hline ACSL 1A & S. seymenliensis & $2.20^{\mathrm{i}}$ & $3.36^{\mathrm{e}}$ & $2.17^{\mathrm{b}}$ \\
\hline ACSL 1B & S. massasporeus & $2.67^{\mathrm{h}}$ & $3.42^{\mathrm{e}}$ & 0.00 \\
\hline ACSL 2 & S. chartreusis & $1.52^{\mathrm{k}}$ & $5.55^{\mathrm{b}}$ & 0.00 \\
\hline ACSL 6 & S. hygroscopicus & $6.42^{\mathrm{a}}$ & $4.00^{\mathrm{e}}$ & $2.60^{\mathrm{a}}$ \\
\hline ACSL 7 & S. galbus & $5.10^{\mathrm{d}}$ & $3.78^{\mathrm{e}}$ & 0.00 \\
\hline ACSL 8 & S. sporocinereus & $5.43^{\mathrm{c}}$ & $3.67^{\mathrm{e}}$ & $1.22^{\mathrm{e}}$ \\
\hline ACSL 12 & K. atroaurantiaca & $4.81^{\mathrm{d}}$ & $2.93^{\mathrm{f}}$ & $1.46^{\mathrm{d}}$ \\
\hline ACSL 13 & S. hygroscopicus & $4.87^{\mathrm{d}}$ & $5.33^{\mathrm{b}}$ & 0.00 \\
\hline ACSL 16A & S. purpeofuscus & 0.00 & $4.17^{\mathrm{d}}$ & 0.00 \\
\hline ACSL 16B & S. galbus & $4.13^{\mathrm{f}}$ & $4.58^{\mathrm{d}}$ & 0.00 \\
\hline ACSL 18A & S. longwoodensis & $3.40^{\mathrm{g}}$ & $3.83^{\mathrm{e}}$ & 0.00 \\
\hline ACSL 18B & S. phaeochromogenes & $2.93^{\mathrm{h}}$ & $4.33^{\mathrm{d}}$ & 0.00 \\
\hline ACSL 22 & S. yunnanensis & $3.15^{\mathrm{g}}$ & 0.00 & 0.00 \\
\hline ACSL 23 & S. indiaensis & $3.22^{\mathrm{g}}$ & $5.13^{\mathrm{c}}$ & 0.00 \\
\hline ACSL 25 & A. rifamycinica & 0.00 & $4.39^{\mathrm{d}}$ & $2.02^{\mathrm{b}}$ \\
\hline ACSL 27A & S. lydicus & $4.31^{\mathrm{f}}$ & 0.00 & $1.66^{\mathrm{c}}$ \\
\hline ACSL 27B & S. corchorusii & $5.00^{\mathrm{d}}$ & $5.50^{\mathrm{b}}$ & 0.00 \\
\hline ACSL 50 & S. sampsonii & $3.13^{\mathrm{g}}$ & $3.93^{\mathrm{e}}$ & 0.00 \\
\hline ACSL 53 & K. paracochleata & $1.65^{\mathrm{k}}$ & $3.44^{\mathrm{e}}$ & 0.00 \\
\hline ACSL 54 & S. sasae & $3.42^{\mathrm{g}}$ & 0.00 & $2.23^{\mathrm{b}}$ \\
\hline ACSL 64A & S. coacervatus & $3.60^{\mathrm{g}}$ & $2.86^{\mathrm{f}}$ & $2.12^{\mathrm{b}}$ \\
\hline ACSL 64B & S. griseoruber & $1.13^{\mathrm{m}}$ & $2.73^{\mathrm{f}}$ & $2.20^{\mathrm{b}}$ \\
\hline ACSL 67 & S. phaeopurpureus & $3.21^{\mathrm{g}}$ & $3.92^{\mathrm{e}}$ & $2.16^{\mathrm{b}}$ \\
\hline ACSL 77 & K. phosalacinea & $2.14^{\mathrm{i}}$ & $4.21^{\mathrm{d}}$ & $1.79^{\mathrm{c}}$ \\
\hline ACSL 80 & S. phaeochromogenes & $1.24^{1}$ & 0.00 & 0.00 \\
\hline ACSL 82 & K. paracochleata & $6.00^{\mathrm{b}}$ & $3.75^{\mathrm{e}}$ & 0.00 \\
\hline ACSL 83 & S. longwoodensis & $2.77^{\mathrm{h}}$ & $4.92^{\mathrm{c}}$ & 0.00 \\
\hline ACSL 85 & S. phaeochromogenes & 0.00 & $4.28^{\mathrm{d}}$ & 0.00 \\
\hline ACSL 91 & S. yunnanensis & $2.71^{\mathrm{h}}$ & 0.00 & 0.00 \\
\hline ACSL 93 & A. echigonensis & 0.00 & 0.00 & 0.00 \\
\hline ACSL 115 & S. thioluteus & $2.37^{\mathrm{i}}$ & 0.00 & 0.00 \\
\hline ACSL 404 & S. chartreusis & $1.98^{\mathrm{j}}$ & $3.90^{\mathrm{e}}$ & $1.52^{\mathrm{d}}$ \\
\hline ACSL 432 & S. novaecaesareae & $4.93^{\mathrm{d}}$ & $3.17^{\mathrm{f}}$ & 0.00 \\
\hline ACSL 448 & S. sioyaensis & $2.61^{\mathrm{h}}$ & 0.00 & 0.00 \\
\hline ACSL 449 & S. yunnanensis & $3.27^{\mathrm{g}}$ & $1.78^{\mathrm{g}}$ & 0.00 \\
\hline ACSL 450 & A. bullii & 0.00 & $3.98^{\mathrm{e}}$ & $1.68^{\mathrm{c}}$ \\
\hline ACSL 453 & S. galbus & $1.96^{\mathrm{j}}$ & 0.00 & 0.00 \\
\hline ACSL 457 & A. pretoriensis & 0.00 & $4.42^{\mathrm{d}}$ & $1.47^{\mathrm{d}}$ \\
\hline ACSL 470 & S. pseudovenezuelae & $4.00^{\mathrm{f}}$ & $4.33^{\mathrm{d}}$ & 0.00 \\
\hline ACSL 485 & S. psammoticus & $4.52^{\mathrm{e}}$ & $4.53^{\mathrm{d}}$ & $1.51^{\mathrm{d}}$ \\
\hline ACSL 490 & A. kentuckyensis & 0.00 & $2.89^{\mathrm{f}}$ & 0.00 \\
\hline ACSL 495 & A. lexingtonensis & 0.00 & $3.20^{\mathrm{f}}$ & 0.00 \\
\hline ACSL 509 & S. deserti & $2.29^{\mathrm{i}}$ & $4.27^{\mathrm{d}}$ & 0.00 \\
\hline ACSL 517 & S. phaeochromogenes & $2.55^{\mathrm{h}}$ & $5.70^{\mathrm{b}}$ & $2.07^{\mathrm{b}}$ \\
\hline ACPM 5 & S. olivochromogenes & $3.29^{\mathrm{g}}$ & $3.58^{\mathrm{e}}$ & $2.07^{\mathrm{b}}$ \\
\hline ACPM 29 & S. scabiei & $5.52^{\mathrm{c}}$ & $5.08^{\mathrm{c}}$ & $2.10^{\mathrm{b}}$ \\
\hline ACPM 31 & S. phaeopurpureus & $3.39^{\mathrm{g}}$ & $4.07^{\mathrm{d}}$ & $2.00^{\mathrm{b}}$ \\
\hline ACPM 38 & S. rishiriensis & $2.83^{\mathrm{h}}$ & $4.47^{\mathrm{d}}$ & 0.00 \\
\hline ACPM 66 & S. Sioyaensis & $4.18^{\mathrm{f}}$ & 0.00 & 0.00 \\
\hline ACPM 346 & S. endophyticus & $3.07^{\mathrm{g}}$ & $3.56^{\mathrm{e}}$ & 0.00 \\
\hline ACPM 363 & S. galbus & $2.62^{\mathrm{h}}$ & $5.06^{\mathrm{C}}$ & $2.27^{\mathrm{b}}$ \\
\hline ACPM 364 & K. viridis & 0.00 & 0.00 & $1.93^{\mathrm{b}}$ \\
\hline ACPM 641 & S. lannensis & $1.90^{\mathrm{j}}$ & $2.75^{\mathrm{f}}$ & $1.37^{\mathrm{e}}$ \\
\hline ACJ 1 & S. ossamyceticus & $5.14^{\mathrm{d}}$ & $4.42^{\mathrm{d}}$ & 0.00 \\
\hline ACJ 17 & S. bangladeshensis & $4.28^{\mathrm{f}}$ & $5.08^{\mathrm{c}}$ & 0.00 \\
\hline ACJ 26 & S. capoamus & $3.29^{\mathrm{g}}$ & $3.83^{\mathrm{e}}$ & $1.75^{\mathrm{c}}$ \\
\hline ACJ 29 & S. galbus & $2.17^{\mathrm{i}}$ & $4.75^{\mathrm{c}}$ & 0.00 \\
\hline ACJ 36 & S. psammoticus & 0.00 & $5.00^{\mathrm{c}}$ & 0.00 \\
\hline ACJ 43 & S. psammoticus & $4.51^{\mathrm{e}}$ & $1.83^{\mathrm{g}}$ & 0.00 \\
\hline ACJ 45 & S. curacoi & $6.44^{\mathrm{a}}$ & $4.17^{\mathrm{d}}$ & $1.13^{\mathrm{e}}$ \\
\hline ACJ 48 & S. chiangmaiensis & 0.00 & $6.56^{\mathrm{a}}$ & 0.00 \\
\hline ACJ 49 & A. rhabdoformis & 0.00 & 0.00 & $1.58^{\mathrm{d}}$ \\
\hline ACJ 51 & S. griseoruber & $3.45^{\mathrm{g}}$ & $4.55^{\mathrm{d}}$ & $2.23^{\mathrm{b}}$ \\
\hline ACJ 52 & S. yaanensis & 0.00 & $4.58^{\mathrm{d}}$ & $2.13^{\mathrm{b}}$ \\
\hline ACJ 53 & S. cyslabdanicus & 0.00 & $6.56^{\mathrm{a}}$ & $2.08^{\mathrm{b}}$ \\
\hline ACJ 66 & S. galbus & $2.03^{\mathrm{j}}$ & $3.55^{\mathrm{e}}$ & 0.00 \\
\hline ACJ 76 & S. yunnanensis & 0.00 & 0.00 & 0.00 \\
\hline ACP 35 & S. laculatispora & $5.70^{\mathrm{c}}$ & $3.67^{\mathrm{e}}$ & 0.00 \\
\hline ACCB 1 & S. variabilis & 0.00 & 0.00 & 0.00 \\
\hline
\end{tabular}

Mean values followed by the same letters do not differ by the Scott-Knott test at 5\% probability.

Genetics and Molecular Research 16 (3): gmr16039703 

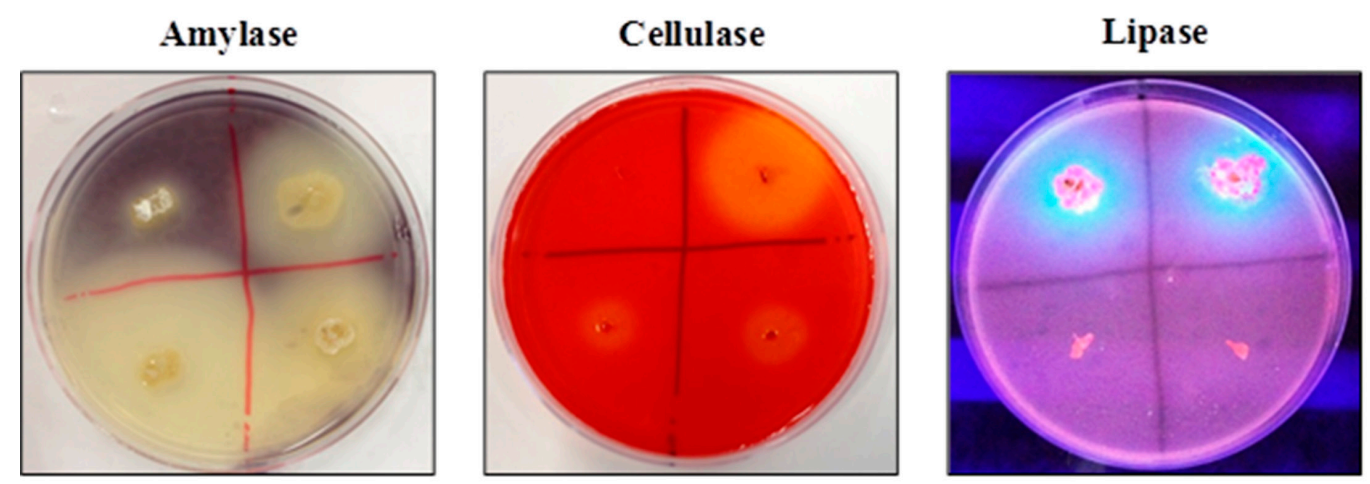

Figure 4. Enzymatic activity of amylase, cellulase, and lipase on agar plate demonstrated by the halo formation around the bacterial colonies.

The results of the EI analysis are shown in Table 4. Sixteen isolates did not produce amylase, and the isolates with $0>\mathrm{EI} \leq 4$ were classified as low and middle potential producers of amylase, and isolates with EI $>4.0$ were identified as the highest enzyme producers. Sixteen isolates of genus Streptomyces and two of Kitasatospora were classified as high amylase producers (EI > 4.0). The isolates ACJ 45 (S. curacoi) and ACSL 6 (S. hygroscopicus) showed the highest $\mathrm{EI}$ (both with $\mathrm{EI}=6.44$ ).

\section{Cellulolytic activity}

The cellulolytic activity was determined by the formation of lighter staining halos (orange) around the colonies against a red background (Figure $4 \mathrm{~b}$ ). The EI for cellulolytic activity varied significantly $(\mathrm{P} \leq 0.05)$ among the Actinobacteria isolates. Fourteen isolates (20.29\%) were not able to form a halo on the CMC plate assay. Therefore, they were considered as non-cellulase producers. Twenty-nine isolates $(42.02 \%)$ were identified as good cellulase producers (EI > 4.0) (Table 4). The isolates ACJ 48 (S. chiangmaiensis) and ACJ 53 (S. cyslabdanicus) showed the highest EI (both with EI = 6.56).

\section{Lipolytic activity}

The lipase activity was measured by the formation of blue staining halos around the colonies against a purple background (Figure $4 \mathrm{c}$ ). The lipase production varied significantly ( $\mathrm{P}$ $\leq 0.05$ ) among the isolates evaluated (Table 4). Fourteen isolates $(20.29 \%)$ showed the highest EI values (EI > 2), and $41(59.42 \%)$ did not show enzymatic activity. The isolate ACSL 6 ( $S$. hygroscopicus $)$ showed the highest EI value $(\mathrm{EI}=2.60)$.

\section{DISCUSSION}

In this study, the analysis of morphological traits and enzyme activity revealed a high level of variability among sixty-nine isolates of Actinobacteria from composting and tropical soils, and molecular sequencing enabled the identification of the isolates at the species level. 


\section{Morphological characterization}

The analysis of morphological characteristics based on valid criteria described in the Bergey's Manual of Systematic Bacteriology (Holt et al., 1994) confirmed all isolates as Actinobacteria. The high morphological diversity and growth habit, characterized by the formation of highly differentiated hyphae and branched aerial mycelium with formation of spores, have been extensively used as valid criteria for identification of genera and species of the phylum Actinobacteria (Ventura et al., 2007; Suneetha et al., 2011; Barka et al., 2015). The grouping of six morphological characters and three enzymatic parameters showed the existence of fifty-seven different morphospecies. Twelve isolates distributed into five different groups and showing the same morphological pattern were considered to represent reisolates of the same sample. In the present study, only the AGA medium was used for bacterial growth. However, it is necessary to point out that the parameter color of the pigment used as a criterion for discriminating among the Actinobacterial isolates may change considerably depending on various cultural factors such medium composition, $\mathrm{pH}$, and temperature of incubation (Holt et al., 1994).

\section{Enzymatic activity}

Enzymes of microbial origin present a great variety of catalytic activities with many applications in various industrial and biotechnological processes, especially in the textile and food industry (Luz et al., 2016). In the present study, the enzymatic activities of amylase, cellulase, and lipase were examined on agar media containing starch, carboxymethylcellulose, and olive oil as substrates, respectively. All but three isolates showed the ability to produce at least one of the three enzymes tested, proving their potential for industrial applications. Members of the three genera identified in the present study are of great importance due to their ability to produce compounds for medical, pharmaceutical, and agricultural purposes.

\section{Amylase activity}

The microbial amylase (EC 3.2.1.1) is among the most relevant classes of enzymes due to the wide range application in industrial biotechnological processes such as processed food, fermentation, and pharmaceutical purposes (de Souza and Oliveira Magalhães, 2010; Adrio and Demain, 2014).

The EI has been used a fast tool for selecting and comparing the enzyme production among different bacterial isolates (Carrim et al., 2006; Castro et al., 2014). Fungaro and Maccheroni (2002) suggested that EI greater than 1.0 are a reliable indicator for the presence of enzymes excreted by microorganisms, while EI $\geq 2.0$ is considered good indicator for potential enzyme production by a bacterium (Lealem and Gashe, 1994). However, while for lipase the variation in EI is minimal ( 2.0), for cellulase and lipase the scale of EI values vary between $>2.0$ to $>6.0$. In the current EI scales, differences of only 0.1 in the scale used to measure enzymatic activity represent a great difference in the potential for enzyme production. In our study, we introduced a new grade of variation in the scale for selecting bacterial species candidates for application in biotechnological processes. In our study, isolates with EI values of $2>\mathrm{EI} \leq 4$ were considered middle producer, and only EI values above 4.0 were considered high producers. Thus, the isolates with EI values $>4$ were selected for further studies aiming at biotechnological applications.

Genetics and Molecular Research 16 (3): gmr16039703 
The production of amylase by microorganisms is very sensitive to incubation temperature. Minotto et al. (2014), performing the enzymatic characterization of endophytic Actinobacteria from tomato plants, observed that the highest EI value recorded for starch degradation was 6.46 when the microorganisms were incubated at $28^{\circ} \mathrm{C}$. Also, results of EI values by Karanja et al. (2010) varied between 3.4 and 5.2 for starch degradation by Streptomyces sp isolates from soil in Kenya. In our study, 32 isolates showed similar results for amylase production with EI values varying between 3.0 and 6.44 using a different medium, and the same incubation temperature $\left(28^{\circ} \mathrm{C}\right)$.

In our study, none of the seven Amycolatopsis isolates showed amylase activity corroborating the study by Ding et al. (2007). In fact, the absence of amylase activity is one important diagnostic feature of this genus of Actinobacteria.

\section{Cellulase activity}

Cellulose, xylan, and lignin (lignocellulose) are the three primary constituents of plant biomass (Yu et al., 2017). These are the most abundant biopolymers in the planet, therefore, the main renewable resources that can be used to produce glucose and proteins, industrial fertilizer, biofuel, and compost (Ramírez and Calzadíaz, 2016). Recently, cellulolytic enzymes of bacterial origin have received special attention of the bioenergy industry due to the higher bacterial growth, sustainability, and environmental impact compared with non-renewable fossil fuel counterparts. However, the industrial-scale breakdown of lignocellulosic plant biomass into simple sugars that can be converted into biofuels is one of the major barriers to the lignocellulosic ethanol production (Lewin et al., 2016).

In Kenya, Karanja et al. (2010) found EI values between 3.4 and 5.2 for starch degradation by Streptomyces sp isolated from soil. In Brazil, Minotto et al. (2014), analyzing Actinobacteria isolated from tomato cultivated in Cerrado soil, and Silva et al. (2015), studying Streptomyces isolated from rhizosphere soil from semi-arid climates, found highest EI values of 4.04 and 6.90, respectively. In our study, 31 isolates of Streptomyces presented EI values between 4.0 and 6.56 indicating their high biotechnological potential uses for largescale industrial enzyme production and composting.

In our study, two Streptomyces (ACP 35 and ACCB 1) from compost did not produce cellulases. Rodrigues (2006) found that $9 \%$ of Streptomyces isolates were not able to degrade cellulose. Considering that the amount of cellulose in the composting process is higher than that available in other soils, the ability to degrade cellulose confers to Streptomyces an important role in the composting processes. Thus, the Streptomyces isolates with the highest EI observed in the present study may be tested for degrading lignocellulose in composting.

\section{Lipase activity}

The enzyme lipase (EC 3.1.1.3) is an important catalyst in biotechnology due to its wide versatility. Lipase can be applied in different industrial processes such as food processing, oils and fats, detergent manufacturing, drug synthesis, cosmetics, and many other products. Microbial lipases have also been used in grease trap waste for the treatment of heavy oils and grease (Bornscheuer, 2002).

The diverse characteristics of lipases produced by microorganisms appear to have evolved to guarantee the fast and efficient access of the microorganism to different sources of

Genetics and Molecular Research 16 (3): gmr16039703 
organic matter (Roveda et al., 2010). Therefore, this group of enzymes is very attractive for industrial applications and recycling processes of lipid-rich compounds (Bornscheuer, 2002).

Despite the knowledge on lipid metabolism in many species of Streptomyces, studies on lipolytic activity in this group are still incipient. Mohamed et al. (2015), evaluating the lipase activity of streptomycetes from soil samples of Taif (Saudi Arabia), detected lipolytic activity in $91.3 \%$ of the isolates. Regarding the EI, Karanja et al. (2010) observed values varying between 3.0 and 4.2 for the lipolytic activity of Streptomyces isolated from soils in Kenya.

In the present study, the highest EI value for lipase was only 2.60, which was lower than the lowest EI value measured by Karanja et al. (2010). These results may be due to the close relationship between the abundance of lipid compounds in the environment and the specific characteristics of the enzymes produced by each microorganism (Gomes et al., 2007). Nowadays, the use of immobilized lipase or whole cell catalysts is one of the most promising methods to produce renewable and environmentally friendly alternative biofuels compared to the non-renewable fossil combustible (Yan et al., 2014).

\section{Molecular characterization}

The molecular data analysis based on the partial sequencing of the 16S rRNA gene enabled the identification of a total of 49 species, being 38 represented by only one isolate and 11 molecular species with more than one strain. Four of the twelve reisolates identified by the morphological traits were confirmed by the molecular analysis.

The three genera of Actinobacteria identified comprise two distinct families of the order Actinomycetales. The genus Amycolatopsis belong to the family Pseudonocardiaceae and the genera Streptomyces and Kitasatospora are classified in the family Streptomycetaceae. However, the three genera are very closely related genetically, and many strains have been misidentified by different authors as belonging to any of these three genera (Ward and Bora, 2015).

Cluster analysis of the morphological and biochemical data grouped the three genera together (data not shown). However, considering only the molecular data, two clusters were observed with the genus Amycolatopsis separated from Streptomyces and Kitasatospora. These results using different criteria reinforce the idea that these subgroups of the phylum Actinobacteria may be recognized as representing a complex of intimately related species, thus difficult to be separated into distinct taxa with the current methods used for species and genus identification. Even studies using detailed molecular data analysis of the 16S rRNA gene, a consensus does not exist about the status of various taxa among systematists of the phylum Actinobacteria. In fact, the variations within the 16S rRNA genes of the Actinobacterial group, even in the variable regions, are insufficient to clarify doubts concerning the identification of species and to estimate the species divergence within a genus or the phylogenetic relationships among genera (Kämpfer et al., 2014); this is the case for the genus Kitasatospora proposed by Omura et al. (1982), which the identity as a valid genus has been changed within years (Zhang et al., 1997; Girard et al., 2014). Bacterial strains identified as belonging to Kitasatospora and Streptomyces exhibit similar lifestyle and morphological traits (Ichikawa et al., 2010). In fact, the differentiation between Kitasatospora and Streptomyces strains is only based on the composition of peptidoglycan in the cell wall. Therefore, the presence of LL-isomer of diaminopimelic acid (DAP) in the aerial mycelia and meso-DAP in the vegetative mycelia of Kitasatospora is the main criterion used to discriminate strains between Kitasatospora and Streptomyces, while the latter display LL-DAP in both the aerial and vegetative mycelia

Genetics and Molecular Research 16 (3): gmr16039703 
(Zhang et al., 1997; Takahashi, 2017). Likewise, representatives of the genus Amycolatopsis and other six genera in the family Pseudonocardiaceae, which was proposed based on the $16 \mathrm{~S}$ rRNA sequence analysis, vary greatly in their morphology and other phenotypic characteristics (Embley et al., 1988). However, the current members of Pseudonocardiaceae contain mesoDAP in their cell wall, and the presence of arabinose and galactose sugars in whole-cell hydrolysates is used as accepted criterion for the diagnosis and species identification in this group (Embley et al., 1988).

Comprehensive comparative studies including protein-coding gene sequences with higher phylogenetic resolution and genome-based studies are needed to clarify the relationships and species delineation within the Streptomycetaceae (Kämpfer et al., 2014).

\section{CONCLUSION}

The ability to degrade several substrates reveals that isolates from the genus Streptomyces, Kitasatospora, and Amycolatopsis of the phylum Actinobacteria have high biotechnological potential uses and may be used in future studies intended to new sources for enzyme production.

\section{Conflicts of interest}

The authors declare no conflict of interest.

\section{ACKNOWLEDGMENTS}

Research supported by Universidade Federal de São João del-Rei (UFSJ), Rede Mineira de Endofíticos and Embrapa Milho e Sorgo (CNPMS). We thank the anonymous reviewers for reviewing this manuscript.

\section{REFERENCES}

Abid I, Mujamammi R and Alkahtani MDF (2016). Antimicrobial activity and molecular identification of Streptomyces strains isolated from Saudi Arabia. J. Environ. Biol. 37: 1225-1230. https://doi.org/10.22438/jeb/37/6/prn.97

Adrio JL and Demain AL (2014). Microbial enzymes: tools for biotechnological processes. Biomolecules 4: 117-139. https://doi.org/10.3390/biom4010117

Altschul SF, Madden TL, Schäffer AA, Zhang J, et al. (1997). Gapped BLAST and PSI-BLAST: a new generation of protein database search programs. Nucleic Acids Res. 25: 3389-3402. https://doi.org/10.1093/nar/25.17.3389

Barka EA, Vatsa P, Sanchez L, Gaveau-Vaillant N, et al. (2015). Taxonomy, physiology, and natural products of Actinobacteria. Microbiol. Mol. Biol. Rev. 80: 1-43. https://doi.org/10.1128/MMBR.00019-15

Battistuzzi FU, Feijao A and Hedges SB (2004). A genomic timescale of prokaryote evolution: insights into the origin of methanogenesis, phototrophy, and the colonization of land. BMC Evol. Biol. 4: 44. https://doi.org/10.1186/1471-2148-4-44

Bornscheuer UT (2002). Microbial carboxyl esterases: classification, properties and application in biocatalysis. FEMS Microbiol. Rev. 26: 73-81. https://doi.org/10.1111/j.1574-6976.2002.tb00599.x

Carrim AJI, Barbosa EC and Vieira JDG (2006). Enzymatic activity of endophytic bacterial isolates of Jacaranda decurrens Cham. (Carobinha-do-campo). Braz. Arch. Biol. Technol. 49: 353-359. https://doi.org/10.1590/S1516$\underline{89132006000400001}$

Castro RA, Quecine MC, Lacava PT, Batista BD, et al. (2014). Isolation and enzyme bioprospection of endophytic bacteria associated with plants of Brazilian mangrove ecosystem. Springerplus 3: 382. https://doi.org/10.1186/2193-1801-3-382

Colen G (2006). Isolamento e seleção de fungos filamentosos produtores de lipases. Tese de doutorado, Universidade Federal de Minas Gerais, Belo Horizonte.

Genetics and Molecular Research 16 (3): gmr16039703 
Coon HJ, Jennison MW and Week OB (1957). Routine tests for the identification of bacteria. In: Manual of Microbiological Methods (Society of American Bacteriologists, ed.). McGraw-Hal, New York, 239-262.

de Souza PM and de Oliveira Magalhães P (2010). Application of microbial $\alpha$-amylase in industry - A review. Braz. J. Microbiol. 41: 850-861. https://doi.org/10.1590/S1517-83822010000400004

Ding L, Hirose T and Yokota A (2007). Amycolatopsis echigonensis sp. nov. and Amycolatopsis niigatensis sp. nov., novel actinomycetes isolated from a filtration substrate. Int. J. Syst. Evol. Microbiol. 57: 1747-1751. https://doi. org/10.1099/ijs.0.64791-0

Embley TM, Smida J and Stackebrandt E (1988). The phylogeny of mycolateless wall chemotype IV actinomycetes and description of Pseudonocardiaceae fam. nov. Syst. Appl. Microbiol. 11: 44-52. https://doi.org/10.1016/S0723$\underline{2020(88) 80047-X}$

Fungaro MHP and Maccheroni W (2002). Melhoramento genético para produção de enzimas aplicadas a Indústria de Alimentos. In: Recursos Genéticos e Melhoramento-Microrganismo (Melo IS, Inglis MCV, Nass LL and Valois ACC, eds.). Embrapa Meio Ambiente, Jaguariúna, 426-453.

Girard G, Willemse J, Zhu H, Claessen D, et al. (2014). Analysis of novel kitasatosporae reveals significant evolutionary changes in conserved developmental genes between Kitasatospora and Streptomyces. Antonie van Leeuwenhoek 106: 365-380. https://doi.org/10.1007/s10482-014-0209-1

Gomes E, Guez MAU, Martin N and Silva R (2007). Enzimas termoestáveis: fontes, produção e aplicação industrial. Quim. Nova 30: 136-145. https://doi.org/10.1590/S0100-40422007000100025

Hardoim PR, van Overbeek LS, Berg G, Pirttilä AM, et al. (2015). The hidden world within plants: ecological and evolutionary considerations for defining functioning of microbial endophytes. Microbiol. Mol. Biol. Rev. 79: 293320. https://doi.org/10.1128/MMBR.00050-14

Hodkinson BP and Lutzoni FA (2009). Microbiotic survey of lichen-associated bacteria reveals a new lineage from the Rhizobiales. Symbiosis 49: 163-180. https://doi.org/10.1007/s13199-009-0049-3

Holt JG, Krieg NR, Sneath PHA, Staley JT, et al. (1994). Bergey's Manual of Determinative Bacteriology. 9th edn. Williams \& Wilkins, Baltimore.

Ichikawa N, Oguchi A, Ikeda H, Ishikawa J, et al. (2010). Genome sequence of Kitasatospora setae NBRC $14216^{\mathrm{T}}$ : an evolutionary snapshot of the family Streptomycetaceae. DNA Res. 17: 393-406. https://doi.org/10.1093/dnares/dsq026

Kämpfer P, Glaeser SP, Parkes L, van Keulen G, et al. (2014). The Family Streptomycetaceae. In: The Prokaryotes (DeLong EF, Lory S, Stackebrandt E and Thompson F, eds.). Springer, Berlin, Heidelberg.

Karanja EN, Boga HI, Muigai AW, Wamunyokoli F, et al. (2010). Optimization of growth conditions and characterization of enzymatic activity of selected novel Streptomyces species from Kenyan soils. Scient. Conf. Proc. 17-30.

Lana UGP, Gomes EA, Silva DD, Costa RV, et al. (2012). Detection and molecular diversity of Pantoea ananatis associated with white spot disease in maize, sorghum and crabgrass in Brazil. J. Phytopathol. 160: 441-448. https:// doi.org/10.1111/j.1439-0434.2012.01924.x

Lealem F and Gashe BA (1994). Amylase production by a Gram-positive bacterium isolated from fermenting tef (Eragrostis tef). J. Appl. Bacteriol. 77: 348-352. https://doi.org/10.1111/j.1365-2672.1994.tb03084.x

Lewin GR, Carlos C, Chevrette MG, Horn HA, et al. (2016). Evolution and ecology of Actinobacteria and their bioenergy applications. Аnпи. Rev. Microbiol. 70: 235-254. https://doi.org/10.1146/annurev-micro-102215-095748

Lewis KJ (1988). Biological control mechanism of the mycoparasitae Phytum oligandum Dreschler. PhD thesis, University of Sheffield, Sheffield, Lima.

Li Q, Chen X, Jiang Y and Jiang C (2016). Morphological identification of Actinobacteria. In: Actinobacteria - Basics and Biotechnological Applications, (Dhanasekaran D and Jiang Y, eds.). Intech Publisher, Rijeka.

Luz BDS, Bicas JL, Sarrouh B and Lofrano RCZ (2016). Bioprospecção de microrganismos produtores de enzimas de interesse industrial realizada no Parque Estadual Serra do Ouro Branco, Brasil. Interbio 10: 13-24.

Minotto E, Milagre LP, Oliveira MT and Van Der Sand ST (2014). Enzyme characterization of endophytic Actinobacteria isolated from tomato plants. J. Adv. Scient. Res. 5: 16-23.

Mohamed HS, Altalhi AD, El-Zahrani GSB and Sadik AS (2015). Enzymatic activities of some streptomycetes isolated from soil at Taif region. Int. J. Curr. Microbiol. Appl. Sci. 4: 19-32.

Omura S, Takahashi Y, Iwai Y and Tanaka H (1982). Kitasatosporia, a new genus of the order Actinomycetales. J. Antibiot. 35: 1013-1019. https://doi.org/10.7164/antibiotics.35.1013

Pridham TG and Lyons Jr AJ (1961). Streptomyces albus (Rossi-Doria) Waksman et Henrici: taxonomic study of strains labeled Streptomyces albus. J. Bacteriol. 81: 431-441.

Ramírez MV and Calzadíaz L (2016). Industrial enzymes and metabolites from Actinobacteria in food and medicine industry. In: Actinobacteria - Basics and Biotechnological Applications (Dhanasekaran D and Jiang Y, eds.). Intech Publisher, Rijeka.

Genetics and Molecular Research 16 (3): gmr16039703 
Rodrigues K (2006). Identificação, produção de antimicrobianos e complexos enzimáticos de isolados de actinomicetos. Dissertação (Mestrado). Universidade Federal do Rio Grande do Sul, Porto Alegre.

Roveda M, Hemkemeier M and Colla LM (2010). Avaliação da produção de lipases por diferentes cepas de microrganismos isolados em efluentes de laticínios por fermentação submersa. Ciênc. Tecnol. Alim. 30: 126-131. https://doi. org $/ 10.1590 / \mathrm{S} 0101-20612010000100019$

Sathya R and Ushadevi T (2014). Industrially important enzymes producing streptomyces species from mangrove sediments. Int J. Pharm. Pharm. Sci. 6: 233-237.

Savitha J, Srividya S, Jagat R, Payal P, et al. (2007). Identification of potential fungal strains for the production of inducible, extracellular and alkalophilic lipase. Afr. J. Biotechnol. 6: 564-568.

Shirling EB and Gottlieb D (1966). Methods for charactrerization of Streptomyces species. Int. J. Syst. Bacteriol. 16: 313 340. https://doi.org/10.1099/00207713-16-3-313

Silva VMA, Brito FAE, Ramos KA, Silva RM, et al. (2015). Atividade enzimática de actinobactérias do semiárido. Rev. Bras. Geofis. 8: 560-572.

Suneetha V, Raj K and Prathusha K (2011). Isolation and identification of Streptomyces ST1 and ST2 strains from Tsunami affected soils: Morphological and biochemical studies. J. Oceanography Marine Sci. 2: 96-101.

Takahashi Y (2017). Genus Kitasatospora, taxonomic features and diversity of secondary metabolites. J. Antibiot. 70: 506-513. https://doi.org/10.1038/ja.2017.8

Tamura K, Peterson D, Peterson N, Stecher G, et al. (2011). MEGA5: molecular evolutionary genetics analysis using maximum likelihood, evolutionary distance, and maximum parsimony methods. Mol. Biol. Evol. 28: 2731-2739. https://doi.org/10.1093/molbev/msr121

Turner S, Pryer KM, Miao VPW and Palmer JD (1999). Investigating deep phylogenetic relationships among cyanobacteria and plastids by small subunit rRNA sequence analysis. J. Eukaryot. Microbiol. 46: 327-338. https:// doi.org/10.1111/j.1550-7408.1999.tb04612.x

Ventura M, Canchaya C, Tauch A, Chandra G, et al. (2007). Genomics of Actinobacteria: tracing the evolutionary history of an ancient phylum. Microbiol. Mol. Biol. Rev. 71: 495-548. https://doi.org/10.1128/MMBR.00005-07

Ward AC and Bora N (2015). The Actinobacteria. In: Practical Handbook of Microbiology (Goldman E and Green LH, eds.). CRC Press, Taylor \& Francis Group, Boca Raton.

Yan J, Zheng X, Du L and Li S (2014). Integrated lipase production and in situ biodiesel synthesis in a recombinant Pichia pastoris yeast: an efficient dual biocatalytic system composed of cell free enzymes and whole cell catalysts. Biotechnol. Biofuels 7: 55. https://doi.org/10.1186/1754-6834-7-55

Yu J, Paterson N, Blamey J and Millan M (2017). Cellulose, xylan and lignin interactions during pyrolysis of lignocellulosic biomass. Fuel 191: 140-149. https://doi.org/10.1016/j.fuel.2016.11.057

Zhang Z, Wang Y and Ruan J (1997). A proposal to revive the genus Kitasatospora (Omura, Takahashi, Iwai, and Tanaka 1982). Int. J. Syst. Bacteriol. 47: 1048-1054. https://doi.org/10.1099/00207713-47-4-1048

Genetics and Molecular Research 16 (3): gmr16039703 